\title{
Fabrication of Multi-layer Self-assembled InAs Quantum Dots for High-Efficiency Solar Cells
}

\author{
Ryuji Oshima*, Hiroyuki Komiyama, Takayuki Hashimoto, Hidemi Shigekawa, and Yoshitaka Okada \\ University of Tsukuba, Institute for Applied Physics, Tsukuba, Ibaraki 305-8573, Japan \\ tel: +81-29-853-6902, fax : +81-29-853-5205, email: bk981495@s.bk.tsukuba.ac.jp
}

\begin{abstract}
We have investigated the characteristics of quantum dot (QD) solar cells with stacked multilayers of self-organized QDs. The GaAs-based $p$-i- $n$ solar cells with 20 stacked InAs QD layers were fabricated by atomic hydrogen-assisted molecular beam epitaxy (H-MBE) on GaAs (001) substrates, and multiple stacking of InAs QDs was achieved by strain-compensation growth technique, in which the tensile strain with respect to GaAs substrate induced by InAs QDs is compensated by a compressive strain induced by GaNAs spacer layers. By controlling the net average lattice strain to a minimum by covering each QD layer with a $40 \mathrm{~nm}$-thick $\mathrm{GaN}_{0.005} \mathrm{As}_{0.995}$ strain compensating layer $(\mathrm{SCL})$, we were successful in obtaining a superior QD stacked structure with no degradation in size uniformity. Further, no dislocations were observed even after 30 layers of stacking, and the area density of QDs amounted to the order of $10^{12} \mathrm{~cm}^{-2}$. The External quantum efficiency characteristics show a spectral response up to $1150 \mathrm{~nm}$. The enhanced absorption performance of the cell in the lower photon energies is attributed to InAs QDs inserted in the $i$-layer.
\end{abstract}

Keywords: Quantum Dots, III-V Semiconductors, Fundamentals

\section{Introduction}

Recently, low dimensional semiconductor structures such as quantum wells (QWs) and quantum dots (QDs) have attracted significant attention as a possible means of exploiting the belowbandgap photons to generate additional photocurrents beyond that corresponding to band-to-band transition in the bulk [1-5]. In QD solar cells, dots should be periodically distributed in space leading to the formation of an intermediate band rather than a multiplicity of discrete levels. Though efficiency enhancements are theoretically predicted in such intermediate band solar cells, which ideally incorporate a three-dimensional (3D) quantum dot superlattice in the active region [6-8], the experimental demonstration of these cells are challenging due to technological difficulties in the fabrication of highly uniform and spatially-ordered QD arrays required in a multiple stack configuration.

The most popular fabrication technique of QDs is to take advantage of spontaneous self-assembly or self-organization mechanism of coherent $3 D$ islanding during growth, known as Stranski-Krastanov (S-K) growth mode in lattice-mismatched epitaxy. In order to achieve these improvements, the fabrication technique to obtain sufficient densities in active region is required. It is well known that stacking is a powerful way of increasing the dot density. However, too many layers of stacking lead to degradation of quality because of accumulation of internal strain with increasing number of QDs layers. Previously, we reported on the preliminary results of $\operatorname{InP}$-based solar cells with 30 multi-layers of self-organized QDs $[9,10]$. In this work, we have carried out basic studies on the characteristics of GaAs-based solar cells with 20 stacked InAs QDs grown on GaAs (001) substrates. Multiple stacking of QDs was achieved by straincompensation growth technique, in which GaNAs alloy was used as a strain compensating spacer layer $[11,12]$.

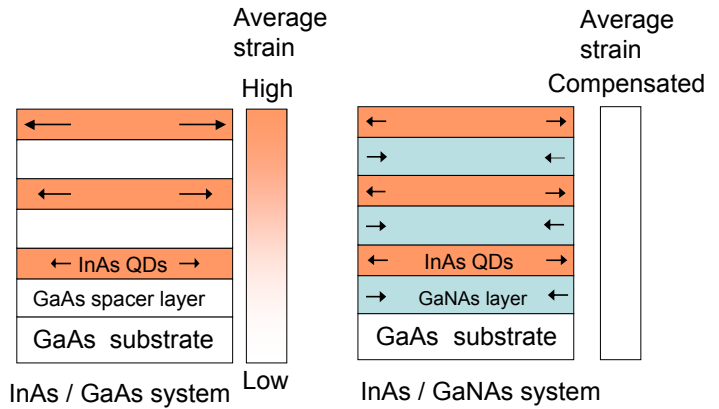

Figure 1. Schematic concept of strain compensation technique in stacking of self-assembled QDs.

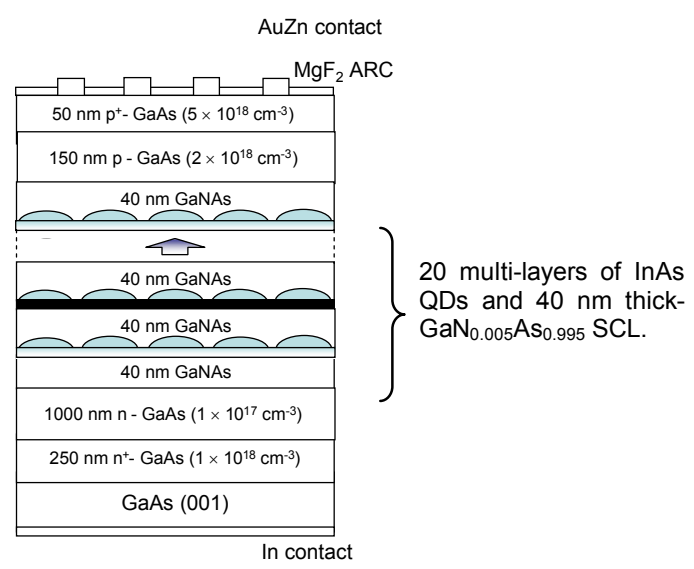

Figure 2. Schematic structure of multi-stacked InAs QD solar cells fabricated in this work. 


\section{Fabrication of multi-stacked Quantum Dots}

For successful implementation of self-assembled QDs to practical devices including solar cells, we first studied a fabrication technique for multi-stacking of InAs QD superlattice structures on GaAs (001) substrates. All growths were done by atomic hydrogen-assisted solid-source molecular beam epitaxy (H-MBE) with a radio frequency (RF) nitrogen plasma source. Figure 1 shows the schematic concept of strain compensation technique for the growth of stacked QD structures. In $\ln (\mathrm{Ga})$ As QDs/GaAs system, the tensile strain with respect to $\mathrm{GaAs}$ substrate generated by growth of $\mathrm{QD}$ layer can not be completely compensated after the growth of GaAs spacer layer, since the lattice constant of spacer layer is the same as substrate. This residual strain affects the formation of QDs in the next stacked layer $[13,14]$. On the other, in $\operatorname{In}(\mathrm{Ga})$ As QDs/GaNAs system, the lattice constant of GaNAs can be controlled to be smaller than GaAs. Therefore, we can satisfy the strain compensation by capping InAs QD layer with a GaNAs layer of suitable lattice constant.

Figure 2 shows the schematic structure of a multilayer stacked QD solar cell fabricated in this work. After the substrate cleaning, $0.25 \mu \mathrm{m}$-thick $n^{+}$-GaAs buffer layer $\left(n=1 \times 10^{18} \mathrm{~cm}^{-3}\right)$, followed by $1 \mu \mathrm{m}$-thick $n$-GaAs base layer $\left(n=1 \times 10^{17} \mathrm{~cm}^{-3}\right)$ were grown at $580^{\circ} \mathrm{C}$. Then, 2.0 monolayers (MLs) of InAs QD layer and $40 \mathrm{~nm}$-thick $\mathrm{GaN}_{0.005} \mathrm{As}_{0.995} \mathrm{SCL}$ were consecutively grown in stack from 10 up to 30 multiple cycles at $480^{\circ} \mathrm{C}$. This stacked configuration was an optimized condition reported elsewhere, in which the tensile strain accommodated around QDs are near perfectly compensated by compressive strain generated by GaNAs layer [11, 12]. The growth rates of QD and spacer layers were 0.1 and $1.2 \mu \mathrm{m} / \mathrm{h}$, respectively. After the growth of intrinsic $Q D$ region, $0.15 \mu \mathrm{m}$-thick $p^{+}$-GaAs emitter layer $\left(p=2 \times 10^{18} \mathrm{~cm}^{-}\right.$ $\left.{ }^{3}\right)$, followed by $50 \mathrm{~nm}$-thick $p^{+}$-GaAs contact layer $(p=$ $5 \times 10^{18} \mathrm{~cm}^{-3}$ ) were grown.

The properties of QDs were studied by using atomic force microscope (AFM), scanning transmission electron microscope (STEM), and photoluminescence (PL) measurements. The PL measurements were done in a temperature-controlled cryostat using a $532 \mathrm{~nm}$ diode pumped solid-state laser at output power of $1 \mathrm{~W} / \mathrm{cm}^{2}$ and liquid-nitrogen cooled InGaAs photodetector. For P-V characterization, AuZn alloy was used for the top contact and In for bottom contact. Standard antireflection coating (ARC) with $\mathrm{MgF}_{2}$ was employed in $0.25 \mathrm{~cm}^{2}$-sized solar cells.

\section{Results and Discussion}

First, AFM images of the topmost QD layer of 30 stacked samples with (a) GaAs spacer layers, and (b) $\mathrm{GaN}_{0.005} \mathrm{As}_{0.995} \mathrm{SCLs}$ prior to embedding in $p-n$ junction are shown in Fig. 3. The sample with no strain compensation in Fig. 3 (a) shows a higher coalesced island density of $3.0 \times 10^{9} \mathrm{~cm}^{-2}$ compared to $4.8 \times 10^{7} \mathrm{~cm}^{-2}$ observed in sample with GaNAs SCL as shown in Fig. 3 (b). Thus, use of GaNAs SCL results in a significantly reduced size fluctuation and better homogeneity. We can calculate a total QD
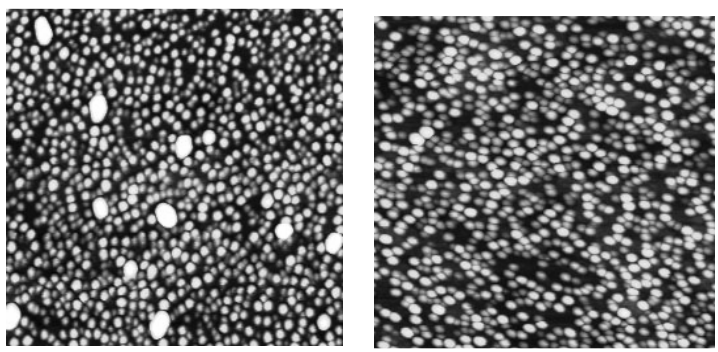

Figure 3. AFM images for the top 30-layer stacked InAs QDs samples embedded with (a) GaAs, and (b) $\mathrm{GaN}_{0.005} \mathrm{As}_{0.995} \mathrm{SCLs}$, respectively. Scan size is $500 \mathrm{~nm} \times 500 \mathrm{~nm}$.

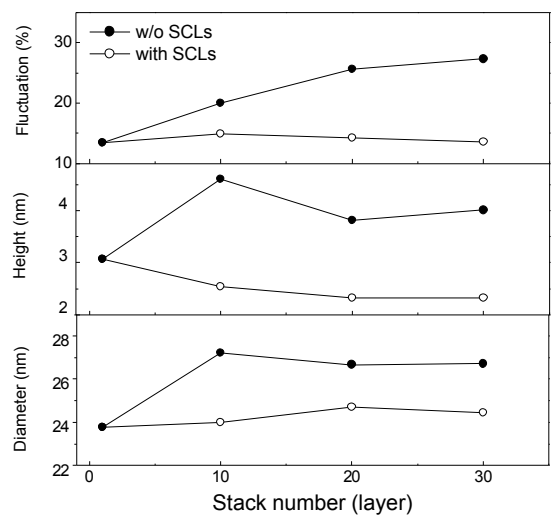

Figure 4. Dependence of average QD lateral size, height, and size fluctuation of stacked InAs QDs on stack number for w/o (closed circles) and with $\mathrm{GaN}_{0.005} \mathrm{As}_{0.995}$ SCLs (open circles), respectively.

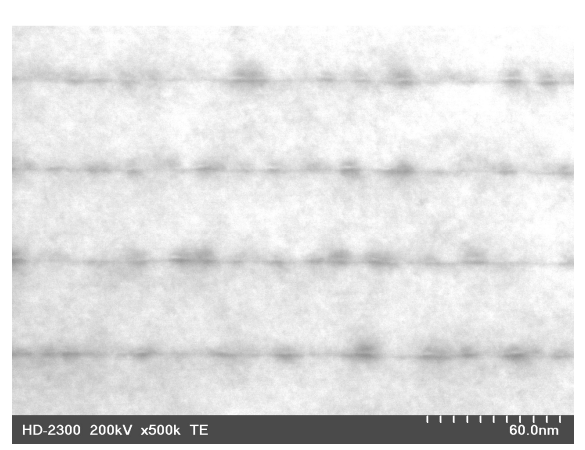

Figure 5. Cross sectional STEM image for 20-layer stacked InAs QDs grown by strain compensation technique.

density of as high as $\sim 3.0 \times 10^{12} \mathrm{~cm}^{-2}$ after 30 layer stacking from Fig. 3 (b). An improved size uniformity of stacked QDs is also evident from the dependence of average QD lateral size, height, and lateral size fluctuation on stack number embedded in GaAs spacer layers and $\mathrm{GaN}_{0.005} \mathrm{As}_{0.995} \mathrm{SCLs}$ as shown in Fig. 4. In the case of samples with GaAs spacer layers, as the stack number increases, the dot size significantly increases and size dispersion becomes degraded as caused by accumulation of internal strain 
due to large lattice mismatch. On the other, QD size in samples with $\mathrm{GaN}_{0.005} \mathrm{As}_{0.995} \mathrm{SCLs}$ are nearly constant even after 30 -layer stacking process. These results suggest that GaNAs SCLs reduce the net strain and also act to suppress the increase of QD size during the stacking process.

Figure 5 shows the cross-sectional STEM image for sample with 20 stacked layers of InAs QDs embedded in $\mathrm{GaN}_{0.005} \mathrm{As}_{0.995} \mathrm{SCLs}$. Only the top portion of the stacked structures is shown. In STEM measurements, it was difficult to obtain information on the strained state of QDs, but the compositional changes due to possible interdiffusion at the heterointerface between QD and spacer layer were studied. Although the actual lattice mismatch at the heterointerface between QD layer and spacer layer becomes larger than that of InAs/GaAs heterointerface, Fig. 5 shows an improved overall crystalline quality with no strain-induced defects and dislocations. It should also be added that interdiffusion of In and/or $\mathrm{N}$ atoms was not observed. In stacking process of QD layers in self-assembled growth, QDs are usually vertically aligned if the spacer layer thickness is relatively thin, typically $10-20 \mathrm{~nm}$ in InAs/GaAs system [9]. However, it is known that the strain field extends over an even longer length. It can be seen from Fig. 5 that the pairing probability of the two neighboring InAs QDs in the vertical direction is still as high as $\sim 48 \%$ even for GaNAs spacer layer thickness of $40 \mathrm{~nm}$. In order to achieve high efficiency QD solar cells, three-dimensional (3D) quantum dot superlattice is desired. However, it is not quite optimal for high-efficiency solar cell applications and adjustments are currently undertaken.

Figure 6 shows the room temperature PL spectra from sample with 20 layers stacked QDs with minimized overall strain by using $\mathrm{GaN}_{0.005} \mathrm{As}_{0.995} \mathrm{SCLs}$ (solid line), and uncontrolled sample with GaAs spacer layers (dashed line), respectively. We observed a single emission peak with a strong PL intensity in the stacked sample, while an $\operatorname{InAs} Q D$ sample with a single layer of dots showed a weak PL emission at room temperature which we measured in a separate experiment. On the other, by burying the QDs with GaNAs SCL, we observe several important features, (1) PL emission wavelength from InAs QDs redshifts from $1120.8 \mathrm{~nm}$ to $1144.9 \mathrm{~nm}$, and (2) full width of half maximum (FWHM) of emission peak is reduced from $65.2 \mathrm{meV}$ to $53.0 \mathrm{meV}$. The redshift of emission wavelength can be explained by the effect of strain compensation and/or reduction of potential barrier of InAs QDs embedded in GaNAs SCLs compared to GaAs spacer layers [16]. The observed reduction of FWHM is clearly attributed to the improvement of size uniformity in growth direction due to the effect of strain compensation as discussed herein.

Figure 7 show the external quantum efficiencies (EQEs) measured for a 20-multilayer QD solar cell with $40 \mathrm{~nm}$-thick $\mathrm{GaN}_{0.005} \mathrm{As}_{0.995}$ strain-compensating spacer layers as a function of reverse bias voltage. It can be seen that a solar cell with embedded InAs QDs into the intrinsic region of GaAs-based $p-i-n$ diode do actually increase the EQE response beyond longer wavelengths and Further, we can see a photoabsorption peak at $910 \mathrm{~nm}$ which corresponds to contribution from GaNAs spacer layer. The

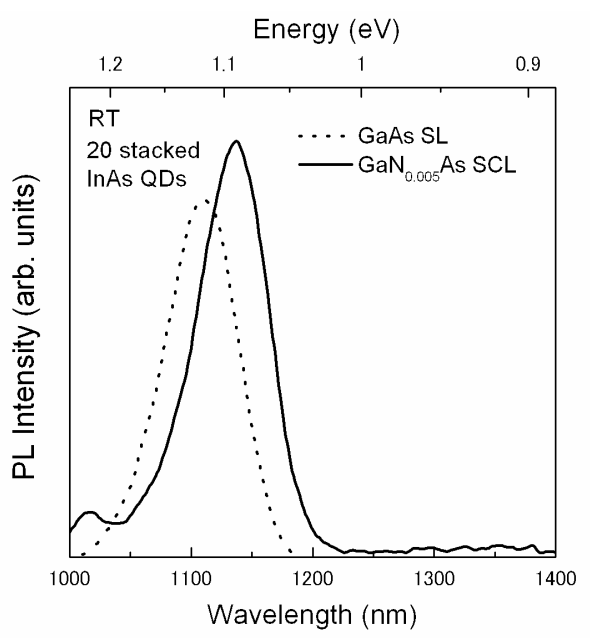

Figure 6. $\mathrm{PL}$ spectra measured at room temperature from 20 stacked layers of InAs QDs embedded in GaAs spacer layers (dashed line), and $\mathrm{GaN}_{0.005} \mathrm{As}_{0.995}$ SCLs (solid line), respectively.

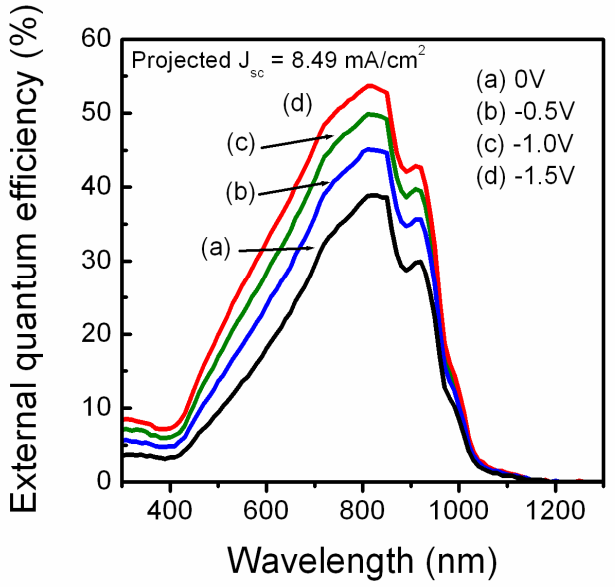

Figure. 7 EQE curves for 20 multi-layers QD solar cell with $40 \mathrm{~nm}$-thick $\mathrm{GaN}_{0.005} \mathrm{As}_{0.995}$ spacer layers as a function of reverse bias voltage. A reverse bias largely improves the photoabsorption in $500 \sim 900 \mathrm{~nm}$ wavelength range, but EQE in the near-infrared region is not much affected.

enhanced absorption performance in the lower photon energies $>\sim 1050 \mathrm{~nm}$ is attributed to contribution from InAs QDs, and this result is in good agreement with $P L$ peak wavelength in Fig. 6. On the other, photoabsorption peak at $1000 \mathrm{~nm}$ may be emission from localized states in GaNAs or excited state in InAs QDs, and these are currently under investigation. It can be further seen that the application of a reverse bias largely affects the photoabsorption in $500 \sim 900$ $\mathrm{nm}$ wavelength range, but EQE in the near-infrared region is not much affected. This suggests that the electron-hole pairs generated in InAs QDs are extracted out of the dots and collected as output current quite efficiently, at least in this sample structure. The optimization of the QD structure leading to the formation of minibands and the cell structure is 
currently underway to further improve the performance.

\section{Summary}

Preliminary studies on the characteristics of GaAs-based QD solar cells with 20 multi-layers of self-assembled InAs quantum dots are presented. The $p-i-n$ solar cells with stacked InAs dot layers were fabricated by atomic $\mathrm{H}$-assisted RF-MBE on GaAs (001) substrates, and multiple stacking of dots was successfully achieved by using GaNAs as strain compensating spacer layer. The uniformity of dot size was $14 \%$ and total dot density on the order of $10^{12}$ $\mathrm{cm}^{-2}$ has been achieved after stacking 20 multi-layers of InAs QDs. No dislocations were generated in samples with $40 \mathrm{~nm}$-thick GaNAs spacer layers. The EQE showed a spectral response up to $1.15 \mu \mathrm{m}$. The enhanced absorption performance of the cell in the lower photon energies is clearly attributed to absorption in InAs QDs in the i-layer. However, further increase in EQE in the near-infrared region is desired for improvement of the QD solar cell, and stacking QD structure seems to play an important role in the overall performance of the cell.

\section{Acknowledgements}

This work was supported in part by the Incorporated Administrative Agency New Energy and Industrial Technology Development Organization (NEDO) under Ministry of Economy, Trade and Industry (METI), Japan, and The Thermal and Electric Energy Technology Foundation. One of the authors (R.O) is supported by Research Fellowships of the Japan Society for the Promotion of Science (JSPS) for Young scientists.

\section{References}

[1] M. Wolf, "Limitations and possibilities for improvement of photovoltaic energy converters", Proc. IRE 48 (1960) 1246.

[2] K. W. J. Barnham and G. Duggan, "A new approach to high-efficiency multi-band-gap solar cells", J. Appl. Phys. 67 (1990) 3490.

[3] N. J. Ekins-Daukes, K. W. J. Barnham, J. P. Connolly, J. S. Roberts, J. C. Clark, G. Hill, M. Mazzer, "Strain-balanced GaAsP/InGaAs quantum well solar cell", Appl. Phys. Lett. 75 (1999) 4195.

[4] Y. Okada, N. Shiotsuka, and T. Takeda, "Potentially modulated multi-quantum wells for high-efficiency solar cell applications", Solar Energy Materials \& Solar Cells 85 (2005) 143.

[5] Y. Okada and N. Shiotsuka, "Fabrication of potentially modulated multi-quantum well solar cells", Proceedings of 31st IEEE Photovoltaic Specialists Conference, Florida (Jan. 2005).

[6] A. J. Nozik, "Quantum dot solar cells", Physica E 14 (2002) 115.

[7] A. Luque and A. Marti, "Increasing the efficiency of ideal solar cells by photon induced transitions at intermediate levels", Phys. Rev. Lett. 78 (1997) 5014.

[8] M. Green, "Multiple band and impurity photovoltaic solar cells: general theory and comparison t tandem solar cells", Prog. Photovolt. Res. Appl. 9 (2001) 137.

[9] K. Akahane, N. Ohtani, Y. Okada, and M. Kawabe, "Fabrication of ultra-high density InAsstacked quantum dots by strain-controlled growth on InP (311)B substrate", J. Crystal Growth 245 (2002) 31.

[10] Y. Okada, N. Shiotsuka, H. Komiyama, K. Akahane, and N. Ohtani, "Multi-Stacking of Highly Uniform Self-Organized Quantum Dots for Solar Cell Applications", Proceedings of 20th European Photovoltaic Solar Energy Conference, June 2005, 1AO.7.6.

[11] R. Oshima, T. Hashimoto, H. Shigekawa, and Y. Okada, "Multiple stacking of self-assembled InAs quantum dots embedded by GaNAs strain compensating layers" J. Appl. Phys. to be published.

[12] R. Oshima, T. Hashimoto, H. Shigekawa, and Y. Okada, "Strain compensation effect on stacked InAs self-assembled quantum dots embedded in GaNAs layers", Material Research Society Symposium Proceedings (Fall 2005), to be published.

[13] G. S. Solomon, J. A. Trezza, A. F. Marshall, and J. S. Harris, Jr., "Vertically aligned and electronically coupled growth induced $\ln A s$ islands in GaAs", Phys. Rev. Lett. 76 (1996) 952.

[14] Q. Xie, A. Madhukar, P. Chen, and N. P. Kobayashi," Vertically Self-Organized InAs Quantum Box Islands on GaAs (100)" Phys. Rev. Lett. 75 (1995) 2542.

[15] R. Oshima, T. Hashimoto, H. Shigekawa, and Y. Okada, "Long wavelength InAs self-assembled quantum dots embedded in GaNAs straincompensating layers" Physica $\mathrm{E}$, in press. 